\title{
Relaxation and Clustering in a Local Search Framework: Application to Linear Placement
}

\author{
SUNG-WOO HUR and JOHN LILLIS* \\ Department of Electrical Engineering and Computer Science, University of Illinois, Chicago, IL, USA
}

(Received 8 September 1999; Revised 4 February 2000)

\begin{abstract}
This paper presents two primary results relevant to physical design problems in CAD/VLSI through a case study of the linear placement problem. First a local search mechanism which incorporates a sophisticated neighborhood operator based on constraint relaxation is proposed. The strategy exhibits many of the desirable features of analytical placement while retaining the flexibility and nondeterminism of local search. The second and orthogonal contribution is in netlist clustering. We characterize local optima in the linear placement problem through a simple visualization tool-the displacement graph. This characterization reveals the relationship between clusters and local optima and motivates a dynamic clustering scheme designed specifically for escaping such local optima. Promising experimental results are reported.
\end{abstract}

Keywords: Linear placement; Local search; Relaxation; Clustering

\section{INTRODUCTION}

Three of the most successful and prominent paradigms for solving physical design problems in CAD/VLSI are constraint relaxation, local search, and netlist clustering. This paper provides new perspectives on each of these topics via a case study in linear placement.

\section{Background}

Constraint relaxation has provided the foundation of numerous university and commercial approaches to the cell placement problem. These techniques are often referred to as "analytical placement" and generally adopt the following or similar philosophy. First, the "slot constraints" of the problem are released resulting in a continuous space optimization problem which can be solved optimally and efficiently. There are several possibilities with respect to the particulars of the continuous formulation-e.g. quadratic placement [4], "linearized" quadratic placement [5], linear programming based relaxations [6] and quadratic placement using spectral methods [7]. A solution to this relaxed formulation results in a physically infeasible placement with cell overlap; this placement is then refined in a topdown manner (typically by recursive partitioning of the layout area) to eventually converge on physically feasible solution. The intuitive appeal of such approaches is that the relaxation solves a true global optimization problem taking into account the entire netlist simultaneously. The methods also tend to be quite computationally efficient. (As an aside, min-cut based placers [8] can also be considered members of this general class. However, the relaxation of slot constraints for min-cut is not continuous-rather many slots are replaced with two (or four in the case of quadrisection [9]) — and the relaxed problem is not solved optimally because of the NPcompleteness of graph partitioning.)

The second paradigm of local search adopts the philosophy of repeated perturbation of a current solution (typically, but not always, physically feasible). A final optimized solution is converged upon via a sequence of such perturbations. Typically, perturbations (or moves) are very simple-e.g. pair-wise exchange. The candidate moves define a neighborhood structure for the problemeach configuration (placement) having a set of neighboring configurations which can be reached in a single move. The intuitive appeal of this kind of approach is multifaceted. First, the entire solution space is reachable via some sequence of moves (assuming a reasonable neighborhood operator). Second, the technique can be made non-deterministic via randomization allowing

*Corresponding author. Tel.: +1-312-355-1317. Fax: +1-312-413-0024. E-mail: jlillis@eecs.uic.edu 
natural exploitation of additional CPU resources (relaxation-based techniques are, by and large, much more deterministic in nature). Further, in the particular case of simulated annealing (SA) [10]-perhaps the most celebrated local search paradigm for cell placementthere are some theoretical results [11] indicating that, with a proper cooling schedule, SA converges to a global optima with probability approaching 1 . Unfortunately, the rate of convergence is not, in general, polynomially bounded. Yet, perhaps the most convincing testimony to the local search paradigm is its remarkable success in practice.

The third paradigm - netlist clustering-can be considered orthogonal to the first two. The motivation for netlist clustering varies significantly from paper to paper. A typical reason for clustering is simply to more effectively deal with huge designs-by pre-processing the netlist and creating a clustered netlist, the problem size becomes more manageable. There are a number of other engineering-oriented reasons for clustering-e.g. in a design with widely varying cell sizes, a clustering step is frequently used to create clusters of roughly equivalent size (thereby enabling the use of cell-oriented algorithms on the clustered netlist). Some recent results in partitioning [12,13] have also successfully applied clustering technology to obtain state of the art results in impressive CPU time. Overall however, it appears that clustering technology is not quite as mature as some other techniques with little consensus on appropriate clustering metrics and on the underlying objectives of clustering itself.

\section{Contributions}

The results in this paper touch on all three of these topics: constraint relaxation, local search and clustering. The contributions are summarized as follows.

- First, we propose a local search mechanism in which the neighborhood operator itself is much more sophisticated and directed than those typically used. The operator is itself based on a linear programming relaxation of the problem where a subset of the cells are "mobile" and the remaining cells are fixed by the current placement. An efficient network flow algorithm is used to solve the linear programming (LP) formulation. Generally speaking, a single move in this scheme is as follows: extract (by some randomized means) a sub-circuit from the netlist; solve the LP relaxation where all cells outside the sub-circuit are at fixed positions as determined by the current placement; heuristically legalize the resulting relaxed placement; evaluate the new placement and accept or reject. The technique adopts a more analytical and global view of the problem while maintaining the desirable features of the local search paradigm. Experimental evidence is presented indicating the potential of such a unification of analytical methods and search-based methods.
- Second, we present studies characterizing local optima in the linear placement problem and their relation to circuit clusters. We have devised a simple visualization tool-the displacement graph-for characterizing the differences between two placements. By studying the differences between known excellent placements and mediocre local optima it is revealed that such local optima typically have successfully found many appropriate clusters of cells, but that these clusters are not globally placed correctly. A local search algorithm working on the flat netlist is unlikely to uncover this structure and as a result it is usually very difficult to improve such solutions (i.e. they are local optima). However, via a simple dynamic clustering strategy based on the current linear placement, such global structure can often be revealed, allowing us to escape the local optima. The key points of the clustering strategy are as follows.

- Clusters should be derived from the current placement. A similar philosophy has been adopted by Saab [3]. The idea is that good placement algorithms do an excellent job of finding good clusters-instead of finding clusters via a pre-process, we let the placer do the work.

- The strategy should be dynamic. While simple strategies based on wiring density seem to identify good clusters in a given placement, it seems unwise to rely on just a few such decisions. As a result, the algorithm repeatedly clusters and flattens the circuit optimizing in each phase via the local search algorithm.

We have dubbed the resulting strategies RBLS ("rebels") for Relaxation Based Local Search and RBLS/C for the dynamic clustering version. These techniques have produced very promising experimental results: new best known results have been found for every circuit in a set of benchmarks used in a recent series of papers on linear placement [1,2]. Additionally, the computational overhead of the techniques is shown to be reasonable.

The remainder of the paper is organized as follows. In the next section, we define some necessary terminologies. In the third section, we present the relaxation based local search algorithm. The fourth section presents our dynamic clustering strategy. The fifth section presents the overall RBLS/C algorithm. The sixth section presents experimental results on the linear placement with MCNC benchmarks, followed by conclusion in seventh section.

\section{PRELIMINARIES}

We model a netlist by a hypergraph $G(V, E)$, where $V$ is a set of cells and $E$ is a set of nets. A hyperedge $e \in E$ is a subset of two or more cells in $V$ (i.e. $e \subseteq V$ ). Each cell 
corresponds to a component of a circuit and each net represents a common signal among its constituent cells.

For a linear placement $P$, let $P[i]$ denote the cell placed in location $i$ and $P^{-1}[v]$ the location of cell $v$ with the assumption that each cell has unit-size. A placement $P$ is legal if $P: N \Rightarrow N$ is one-to-one function. Let $e_{i}$ be a set of cells which are connected to a net $i$. Then, the length of net $i$, len $\left(e_{i}\right)$, is defined as

$$
\operatorname{len}\left(e_{i}\right)=\max _{u, v \in e_{i}}\left|P^{-1}[u]-P^{-1}[v]\right|
$$

and the linear placement problem as

$$
\min \sum_{i=1}^{|E|} \operatorname{len}\left(e_{i}\right) \quad \text { over all legal placement } P \text {. }
$$

\section{RELAXATION-BASED LOCAL SEARCH}

\section{Overview}

The top-level local search strategy used in RBLS is quite traditional. From the current solution we sample a neighboring solution and move to that solution if the objective (wire length) is improved. If no improvement is seen for $k$ (a given parameter) consecutive moves, the search terminates. The novelty of RBLS is in how we generate neighboring solutions. This process is summarized as follows:

- Sub-circuit extraction: Given a parameter $m$, extract a sub-circuit $M(\subseteq V)$ where $|M|=m$. $M$ will be called the set of "mobile nodes". From $M$ we determine the fixed node set $F$ : the set of nodes in $(V-M)$ which are directly connected to a member of $M$ via some net. Extracted sub-circuit consists of node set $F \cup M$ and net set $E^{\prime}$ induced by $M$, i.e. $G^{\prime}=\left(F \cup M, E^{\prime}\right)$.

- Optimal relaxed placement: Optimally place each member of $M$ ignoring slot constraints under a linear programming relaxation of the problem. Note that the relative order of cells in $F$ influences this solution.

- Placement legalization: Resolve cell overlap to obtain a physically feasible placement.

Each of these steps is detailed in the following subsections.

\section{Sub-circuit Extraction}

To extract a sub-circuit $G^{\prime}=\left(V^{\prime}, E^{\prime}\right)$, where $V^{\prime}=F \cup M$, we extract $M$ first. The simplest method for extracting a subset $M$ would be a random selection. However, such a simple scheme would result in many disconnected components and the resulting optimization problem does not capture much of the interaction between cells.
Therefore, we have adopted the following randomized scheme which produces a set of connected components. Experiments have shown this scheme to be superior to simple random selection. First, given a parameter $m$, mobile nodes are selected as follows:

1. $M \leftarrow \varnothing / *$ mobile node set $* /$

2. $C \leftarrow \varnothing / *$ Set $C$ has candidate nodes for mobile nodes $* /$

3. Extract a node $v \in V M$ at random and $M \leftarrow M \cup\{v\}$

4. $A \leftarrow\{u \in e \mid v \in e, \forall e \in E\} / *$ set of nodes adjacent to $v * 1$

5. $C \leftarrow C \cup A M$

6. If $|C|=0$ go to step 3

7. Extract a node $v \in C$ at random and $M \leftarrow M \cup\{v\}$

8. Repeat step 4-7 while $|M|<m$

Once $M$ is extracted, we determine the fixed node set $F$ and the "active nets" $E^{\prime}$ (all nets which influence the relaxed placement problem for $M$ ) as follows: $E^{\prime}=$ $\left\{e_{i} \mid\right.$ for some node $\left.u \in M, u \in e_{i}\right\} . \quad F=\left\{v \mid v \in e_{i} M\right.$, where $e_{i} \in E^{\prime}$, and $v$ is either left- or right-extreme node of $e_{i}$ in the current placement $P$ \}.

Figure 1, shows an example of extracted sub-circuit and current placement $P$.

\section{LP-formulation of a relaxed Placement}

Given a set of mobile nodes $M$, we can derive a simple linear program for optimally placing $M$. Such a linear program will produce an $x$-coordinate $x_{v}$ for each mobile node $v \in M$. The LP is of course influenced by the locations of the fixed nodes $F$; let $X_{v}$ be the location of a node $v \in F$ in a given feasible placement $P$, i.e. $X_{v}=$ $P^{-1}[v]$. Then the LP relaxation can be stated as follows.

$$
\begin{gathered}
\min \sum_{e \in E^{\prime}}\left(r_{e}-l_{e}\right) s . t . \\
l_{e} \leq x_{v} \leq r_{e}, \forall v \in e, \\
x_{v}=X_{v}, \forall v \in F
\end{gathered}
$$

The dummy variables $r_{e}$ and $l_{e}$ in the formulation give the leftmost and rightmost ends of net $e$.

To illustrate the relaxation process, suppose we have a given legal placement $P$ and sub-circuit as shown in Fig. 1.

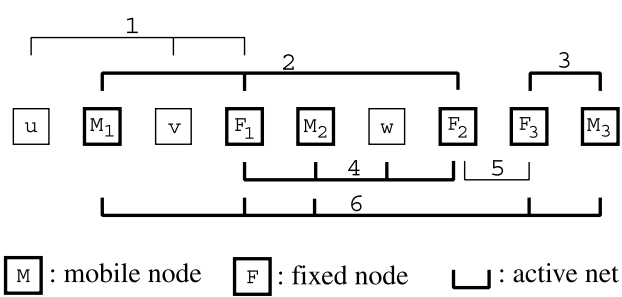

FIGURE 1 An example of the current placement $P$ and a sub-circuit extracted from $P$. Total wire length of $P$ is 20 . 


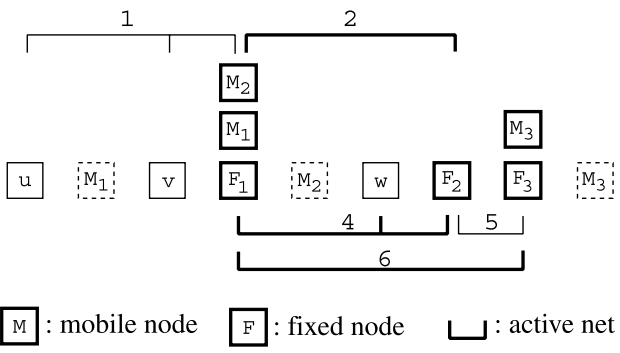

FIGURE 2 An example of an optimal relaxed placement, which is induced by the sub-circuit shown in Fig. 1 . Net 3 is not shown in the placement since its wire length becomes 0 . The total wire length is 14 .

Figure 2 shows a possible solution of LP (optimal solutions not being necessarily unique).

In early experiments, a public domain LP-solver $\dagger$ was used and it indeed proved to be an unacceptable bottleneck. Fortunately, it was discovered that the solution of the linear program could be obtained very efficiently by using network flow techniques presented next.

\section{Network Flow Based Algorithm}

We utilize a simple network flow based algorithm for solving the LP relaxation used in our neighborhood operator. The algorithm is a refinement of Picard and Ratliff's solution to the Rectilinear Distance Facilities Locations Problem [14]. The algorithm iteratively finds minimum cuts from left to right which assign mobile nodes to bins formed by each of the fixed nodes. The resulting placement is an optimal solution to the LP formulation. The algorithm has also proved to be very efficient in practice.

Given an extracted sub-graph $G^{\prime}=\left(V^{\prime}, E^{\prime}\right)$ and the current placement $P$, we use $f_{i}(1 \leq i \leq|F|)$ to denote the $i$ th fixed node from the left, and assume all the nodes in $F$ are arranged according to their $x$-coordinates i.e. $P^{-1}\left[f_{i}\right]<P^{-1}\left[f_{j}\right]$ if $i<j$.

As illustrated in Fig. 3, we add additional nodes, source $s$ and sink $t$ and additional edges of capacity $\infty$ which connect fixed nodes and source/sink. We assume every net is 2-pin net and represent two opposite directed edges as a single undirected edge. For some $k(1 \leq k \leq|F|)$, every fixed node $f_{i}(1 \leq i \leq k)$ is connected to $s$ via edge $\left\langle s, f_{i}\right\rangle$ and every fixed node $f_{j}(k<j \leq|F|)$ to $t$ via edge $\left\langle f_{j}, t\right\rangle$ as shown in Fig. 3.

A min-cut is a bipartitioning $\left(A_{k}, A_{k}\right)$ of $G^{\prime}$ with $s \in A_{k}$, $t \in A_{k}$ such that the cutsize $c\left(A_{k}, A_{k}\right)=\sum_{u \in A_{k}, v \in A_{k}} c\langle u, v\rangle$ is minimized, where $c\langle u, v\rangle$ is the capacity of edge $\langle u, v\rangle$.

Figure 4 shows the algorithm relaxed placement (RP). In the algorithm, the set Left is used to keep the mobile nodes of the current min-cut. We change directed edges between fixed nodes and source/sink one by one from the left to right. At step $i$, a new set of mobile to be placed with fixed node $f_{i}$ is identified and added to the set Left.

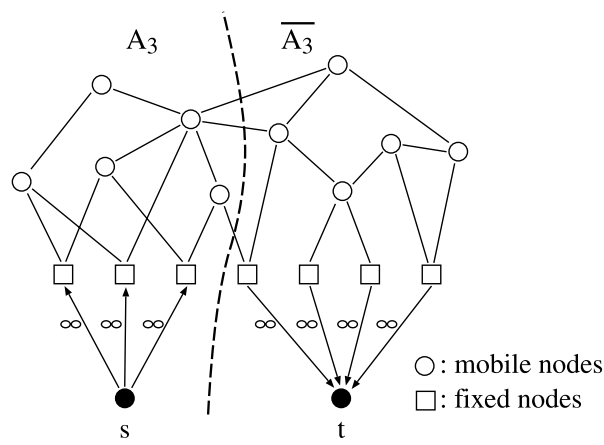

FIGURE 3 An example of a min-cut.

The efficiency of the algorithm is improved by maintaining residual capacities from one step to the next. As a result we are able to avoid re-computation of many augmenting paths.

Figure 5 illustrates an example of the set New after executing one iteration from the state shown in. Mobile nodes $u$ and $v$ become new members of New after modifying connectivity of the fixed node $w$.

The correctness of the algorithm is given by the following theorem and proof sketch (see Ref. [14] for a more detailed presentation).

\section{THEOREM 1 The algorithm RP finds an optimal relaxed placement.}

Proof Sketch: For simplicity, assume w.l.o.g. that all nets have only two pins. First, a simple argument shows that there always exists a solution to the LP in which all mobile nodes are coincident with fixed nodes (as in the case of the placement produced by our network flow algorithm); call such a solution a binned solution (each mobile node belongs to a bin defined by a fixed node); any mobile node not coincident with a fixed node is unbinned. We can transform an unbinned optimal placement into a binned one as follows. Let $v^{*}$ be a maximal set of one or more coincident unbinned nodes where no other unbinned nodes are placed between it and some fixed node $f_{i}$. The number of edges connecting to $v^{*}$ from the left must equal that on the right-otherwise, moving $\epsilon$ units in one direction would reduce the wire length violating the optimality assumption. Thus, $v^{*}$ can be moved freely to $f_{i}$ without increasing the wire length. This process is repeated until all nodes are binned.

Now what remains to be shown is that the binned placement found by the network flow algorithm is optimal. This follows almost directly from the construction. Consider the cut formed at the $i$ th step of the algorithm. The result is a partition $\left(A_{i}, \bar{A}_{i}\right)$ where $f_{1}, \ldots, f_{i} \in A_{i}$ and $f_{i+1}, \ldots, f_{|F|} \in \bar{A}_{i}$. The resulting cutsize $c\left(A_{i}, \bar{A}_{i}\right)$ is the number of wires which pass between $f_{i}$ and $f_{i+1}$. Since the cut is minimum, there can be no other linear placement with fewer than $c\left(A_{i}, \bar{A}_{i}\right)$ wires between $f_{i}$ and $f_{i+1}$. Since the algorithm achieves this lower-bound between each $f_{i}$

\footnotetext{
${ }^{\dagger} \mathrm{ftp}: / / f t p . e s . e l e . t u e . n l / p u b / l p \_s o l v e$.
} 


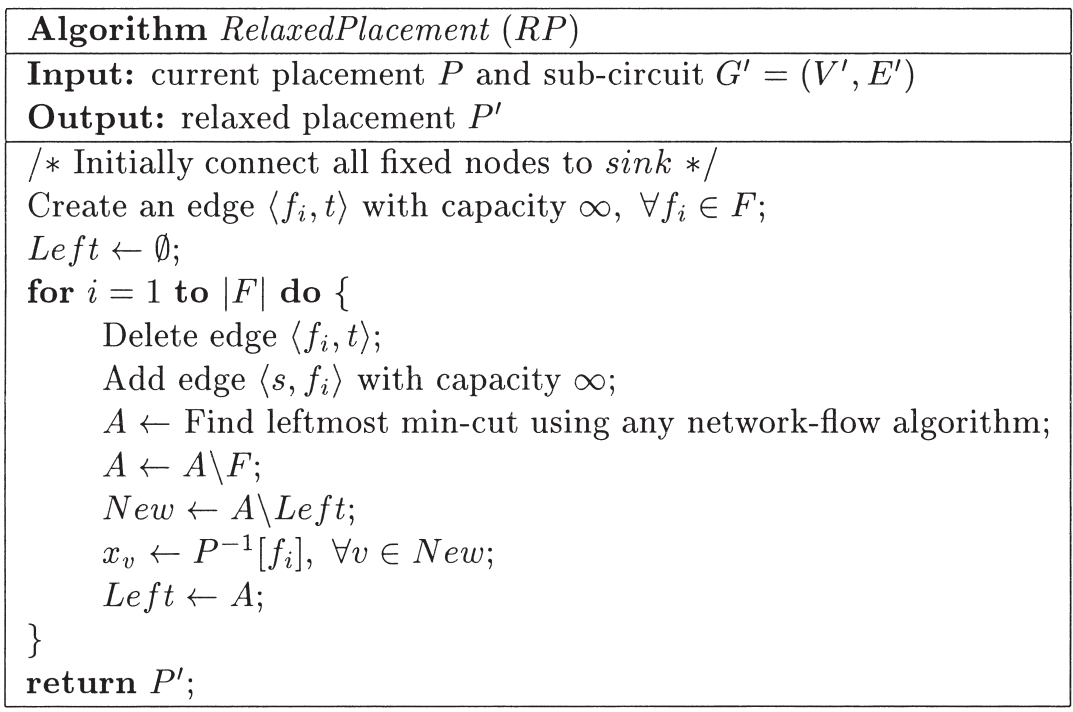

FIGURE 4 The algorithm Relaxed Placement (RP). All the current residual capacities are kept for next iteration in the loop.

and $f_{i+1}$, we conclude that the overall wire length must be minimum.

As an aside, we note that such a binned placement is not necessarily the best in the sense that it may be more difficult to effectively legalize than other optimal solutions. Thus, techniques for finding optimal relaxed placements with more even distribution are worth studying (e.g. via finding equivalent cuts and exploiting node mobility).

We use standard constructions $[15,16]$ to model the hypergraph as a directed graph $\tilde{G}$. The hypergraph to digraph construction is illustrated in Fig. 6 and is summarized as follows.

- Every 2-pin net $e_{i}=\{u, v\}$ in $G$ is associated with two directed edges $\langle u, v\rangle$ and $\langle v, u\rangle$ in $\tilde{G}$ with each having capacity 1 .

- Every multi-pin net $e_{i}$ in $G$ introduces two dummy nodes $a, b$ with an edge $\langle a, b\rangle$ with capacity 1 and directed edges $\langle u, a\rangle$ and $\langle b, u\rangle$ for every $u \in e_{i}$ with capacity $\infty$.

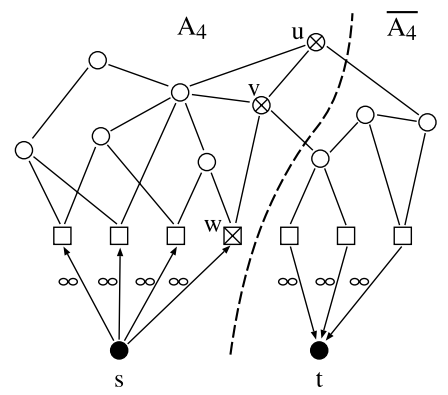

FIGURE 5 A new min-cut after executing one iteration of RP from the state shown in. Mobile nodes $u$ and $v$ become members of "New".
In Fig. 7 we show an example of an optimal relaxed placement which is found by the algorithm RP and induced by the sub-circuit shown in Fig. 1. Note that the two optimal relaxed placements-one is shown in Fig. 2 and the other in Fig. 7-have the same cost.

Empirical results have shown remarkable speedups versus the LP-solver. For example, an instance with 5000 mobile nodes and roughly 1000 fixed nodes can be solved in less than 8 seconds on a $167 \mathrm{MHz}$ Sun Ultra-Sparc 1 while the LP-solver takes about $55 \mathrm{~min}$.

\section{Placement Legalization}

A central problem in relaxation-based methods is the resolution of cell overlaps or legalization. A relaxed placement may result in empty spaces and overlapping nodes that should be resolved to get a physically feasible placement.

Our legalization scheme is quite simple. We use a force value to get a relative order among coincident nodes. Let $S(\subseteq M)$ be a set of coincident nodes and $n_{i}$ a set of nets adjacent to node $i$. Suppose the position for each node in $S$ in the relaxed placement is $x_{s}$. Let $l_{e}$ be the left extreme end of a net $e$ and $L_{i}$ be a subset of $n_{i}$ such that $L_{i}=$ $\left\{e \mid e \in n_{i}\right.$ and $\left.l_{e}<x_{s}\right\}$. Similarly, let $r_{e}$ be the right extreme end of a net $e$ and $R_{i}$ be a subset of $n_{i}$ such that $R_{i}=\left\{e \mid e \in n_{i}\right.$ and $\left.r_{e}>x_{s}\right\}$. Then, the force value $d_{i}$ for node $i$ in $S$ is computed as $d_{i}=\left|R_{i}\right|-\left|L_{i}\right|$. Figure 8shows an example of force values when $|S|=4$.

Using the force values, we decide the relative order of nodes in $S$, e.g. the greater force value a node has the greater $x$-coordinate will be assigned to it. For the nodes having the same force value we randomly rearrange them. After determining the relative order of nodes in $S$, we place them at the position of the associated fixed node while moving other nodes keeping their relative order. 


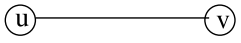

A 2-pin net

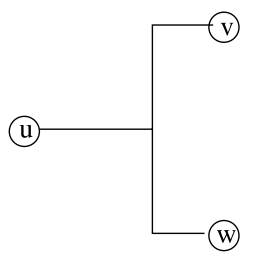

A multi-pin net

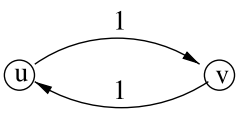

The corresponding net

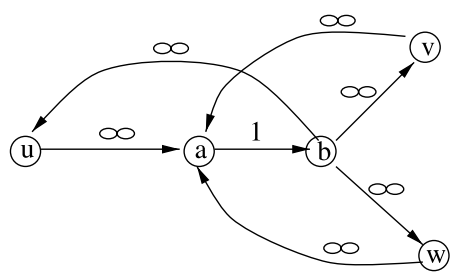

The corresponding net

FIGURE 6 Net modeling.

Figure 9 shows the corresponding legalized placements induced by the relaxed placement which is obtained by the RP algorithm as shown in Fig. 7.

\section{DYNAMIC CLUSTERING}

Effective methods for escaping local optima are essential in most successful local search schemes. We propose a simple dynamic clustering technique for precisely this purpose. We will use the notion of local optima somewhat loosely to indicate a solution which has not improved significantly in a "long time". To the best of our knowledge, this intimate relationship between clusters and local optima has not previously been studied in the literature.

Our clustering technique was motivated by an effort to characterize local optima in the linear placement problem via a simple visualization tool we call a displacement graph. Suppose $P_{g}$ is a relatively good placement and $P_{b}$ a mediocre one. Also assume that each has converged to local optima. If we consider $P_{g}$ as a reference placement, displacement of $P_{b}$ for each location $i$ with respect to $P_{g}$ is

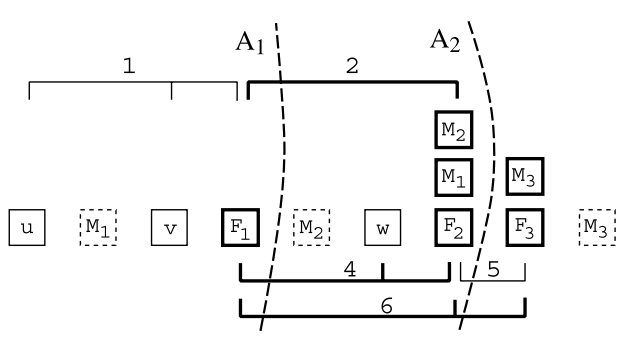

$\mathrm{M}$ : mobile node $\mathrm{F}$ : fixed node $\sqcup$ : active net

FIGURE 7 An example of an optimal relaxed placement found by the algorithm RP. This is induced by the sub-circuit shown in Fig. 1. Net 3 is not shown in the placement since its wire length becomes 0 . The total wire length is 14 . defined as

$$
D[i]=P_{g}^{-1}\left[P_{b}[i]\right]-i .
$$

Figure 10 shows an example of displacement. A positive value indicates that the cell at that position in $P_{g}$ is placed to the left in $P_{b}$; a negative value to the right.

Figure 11 shows a small range of displacement graph so details can be seen where we plot $i$ versus $D[i]$. It is striking how we can easily identify "plateaus" in the plot with sharp transitions between plateaus. A plateau indicates that the bad placement seems to correctly group many cells into the right clusters, but that these clusters are not correctly placed in the global scheme of things. This seems to be a signature of a local optimumi.e. it appears that almost any neighborhood structure would have a hard time uncovering this global structure. This has led us to a dynamic clustering technique for escaping local optima.

Suppose a placement is converged to a local optima. We know that the placement might have good clusters which correspond to plateaus in a displacement graph (versus some hypothetical "good" solution). The goal will be to identify these plateaus dynamically. If this can be done,

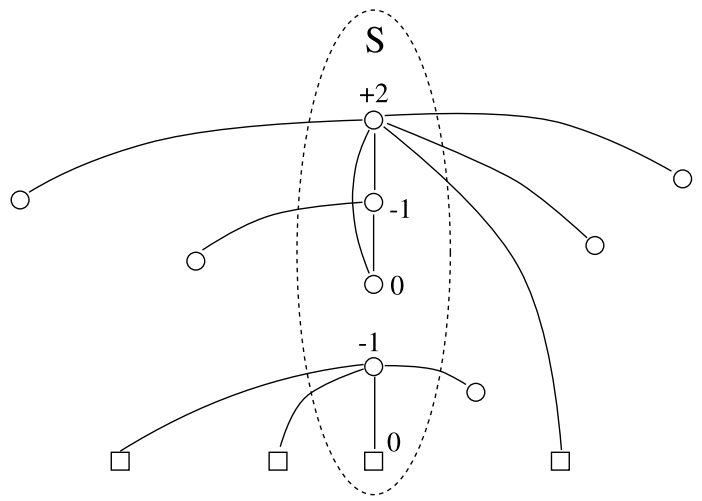

FIGURE 8 An example of force values for coincident nodes. 


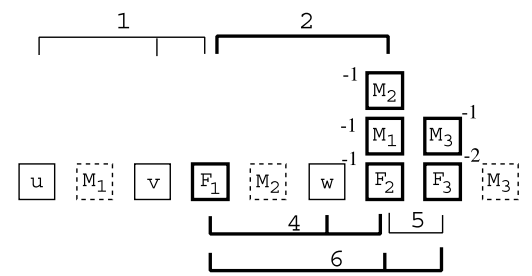

Relaxed placement obtained by the algorithm RP

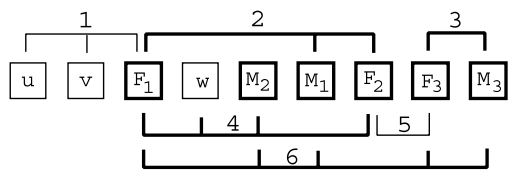

The corresponding legalized placement

FIGURE 9 An example of a legalized placement induced by the relaxed placement which is shown in Fig. 7. The force value for each coincident node is shown on the shoulder. The legalized placement has wire length of 18 resulting in $10 \%$ improvement.

we will have an effective tool for escaping local optima. Said another way, improvement in solution quality in the clustered circuit's search space becomes more likely as is shown experimentally later in this section. Of course, since a good reference placement is not available to help us identify plateaus as clusters, we must resort to other schemes to heuristically estimate cluster boundaries. A simple conjecture is that there is a correlation between transitions in wiring density (number of wires) in the bad placement and the edges of plateaus. Our experiments indicate some truth to this conjecture and such a heuristic has become the basis for our clustering strategy described next.

Figure 12 shows a portion of the correlation between the edges of plateaus and their corresponding density values which supports the conjecture. Almost every edges of plateaus match with the locally low values in density. Of course, it is unreasonable to expect complete correlation but density values do appear to be a good first order guide. The fact that the clustering strategy is imperfect (as is, we argue, any similar clustering strategy), is offset by the dynamic nature of the clustering strategy-we repeatedly cluster and flatten always based on the current placement.

We derive clusters from the current placement $P$ as follows: given two parameters $L$ and $U$, and the current placement $P$, scanning $P$ from the left to the right it clusters a block of nodes using the density values such that each block size is between $L$ and $U$ and the boundary of the next block is the point at which the density value is the smallest among $d[p+L] \cdots d[p+U]$, where $d[i]$ is the density value of position $i$ and $p$ is the boundary position of the previous cluster.

Using the clustering algorithm we generate a clustered circuit and a clustered placement $P_{c}$ based on $P$. Each cell in the clustered circuit is considered to have unit-size. Given $P_{c}$, the relaxation based local search algorithm of "Relaxation-based local search" section is applied until

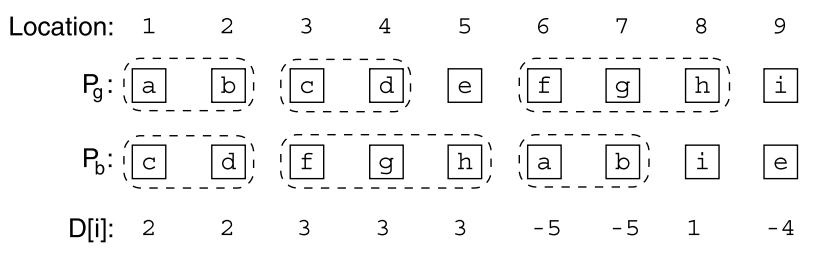

FIGURE 10 An example of displacement. convergence criteria is met and then it is flattened. While flattening $P_{c}$, each clustered node is examined to see whether the reversed order may result in a better solution, and if so, the block of clustered node is placed in reverse order in the flat placement.

Note that since the clustering strategy is dynamic and clustering is always based on the current solution, one clustered circuit is different from previous and subsequent clusterings of the netlist.

Figure 13 illustrates the effectiveness of the technique in escaping local optima. The figure shows a snapshot of a run of the algorithm (CPU time versus wire length). Up to the first transition point (a near local optimum) the placement was optimized in flat mode. At this point, we cluster the circuit and continue the run on the clustered circuit. Wire length in the graph always gives the wire length of the flat placement implied by the clustered placement. After clustering, the result shows a sharp reduction in wire length. When there seems no significant improvement on clustered circuit, we flatten it and continue to optimize the flat circuit. This cycle is repeated and shows the remarkable effectiveness of the dynamic clustering strategy.

\section{OVERALL ALGORITHM}

In Fig. 14, we show the overall procedure of our relaxation-based local search algorithm with dynamic clustering, RBLS/C. The algorithm can be summarized as follows. First, an initial placement is generated. We then repeatedly cluster and flatten the circuit, performing local search on both flat and clustered solutions. This is done until some convergence criteria are met. Throughout the algorithm, there is also some freedom in assigning the control variables (e.g. sub-circuit size for RBLS and target cluster size).

We use several control variables to enable the algorithm to find high quality solution more efficiently. Values for them are changed based on either the circuit size, solution quality, the number of iterations, or the netlist type (flat or clustered).

The control variables $m$ and $m_{c}$ are used to determine the sub-circuit size of flat and clustered netlists, respectively. Initial size of a sub-circuit $\left(i_{0}\right.$ and $i_{1}$ are used for each type) is proportional to the circuit size and it 


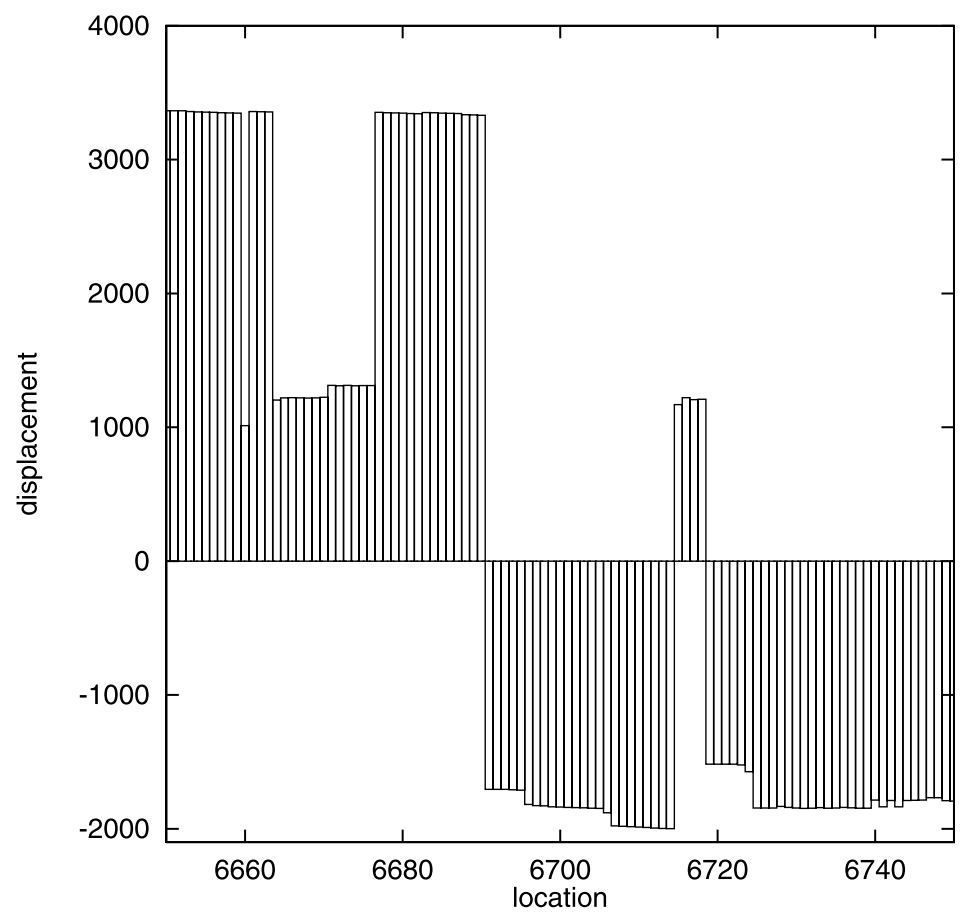

FIGURE 11 Displacement graph obtained from two local optima placements for circuit s38584. Mediocre solution has wire length of 1,756,332, while good reference placement has wire length of 1,253,972.

is gradually decreased to a lower bound of $10 . I_{\max }$ and $I_{\max , c}$ are used for the maximum number of moves in the local search algorithm without improvement for flat and clustered circuits, respectively. Those values are monotonically increasing by setting $\alpha>1$. As solution quality improves, experiments show that selecting smaller subcircuits and giving more chances to explore neighbors is more effective. $L$ and $U$ are used to determine a target block size for clustering. The average block size in clustered circuits is dynamically changed by varying $L$ and $U$. $L$ increases up to $\omega$ in the first phase and decreases to 1 in next phase. We alternate increasing and decreasing phases. By this way we have more chances to find globally right locations for different clusters on different placements.

\section{EXPERIMENTAL RESULTS}

We have implemented the RBLS/C algorithm and tested it on a standard set of benchmarks on a $167 \mathrm{MHz}$ Sun UltraSparc 1.

As described, there are a number of control parameters to the RBLS/C algorithm. While the strategy for initializing these values is not central to our contributions, for completeness, we describe our current strategy in the following. The strategy is based on experimental experience and intuition. The strategy may evolve over time as we gain more insight into their effects; nevertheless, the current approach does seem reasonable. For the initial sub-circuit size we set $i_{0}$ to $0.8 *|V|$ and $i_{1}$ to $0.02 *|V|$. We set $\gamma$ to approximately $0.25-0.3$ and $\delta$ to
0.4-0.6. We use 10 as a lower bound on both $m$ and $m_{c}$. We set $i_{2}$ - the initial value for $I_{\max }$ - to 5 . We set $\alpha$ to 3 , i.e. for a clustered circuit the local search algorithm will allow three times as many consecutive failed moves before exiting. This strategy seems effective since the CPU time required to visit a neighbor in the clustered circuit is generally much less than in the flat circuit. Further, since the wire-length improvement per move in the clustered circuit tends to be greater than in the flat circuit, the benefit of increasing $I_{\max }$ in this way seems to outweigh the additional CPU time. $L$ is initially assigned 2 via $i_{3}$. By setting $\mu=2$ it is doubled up to $\omega$, which is usually assigned 128 , in increasing phase and cut by half in deceasing phase per each loop. To determine the upper bound on cluster size $U$, we set $\beta$ to 1.5-2.0. When we set $\beta$ to a number $>2.0$, the range of each block size of course becomes wider. Since one block is considered as a unitsize cell in clustered circuit a wider range of cluster sizes results in more error in the wire length calculation (vs. the induced flat placement). For this reason, we maintain a fairly narrow range of acceptable cluster sizes. We set $\epsilon$ to $1.0 \times 10^{-5}$.

Lastly, to construct the initial placement we use the Max-Adjacency Ordering method [17]. For certain circuits (e.g. s35932) the initial placement obtained by this method is highly influential on the final solution quality while for most others the initial placement had little influence on the final solution quality. The influence of initial placement on final solution quality is a topic of on-going research.

We have run experiments on the same set of circuits tested with Sato's Simulated Quenching method (SQ) [2] 

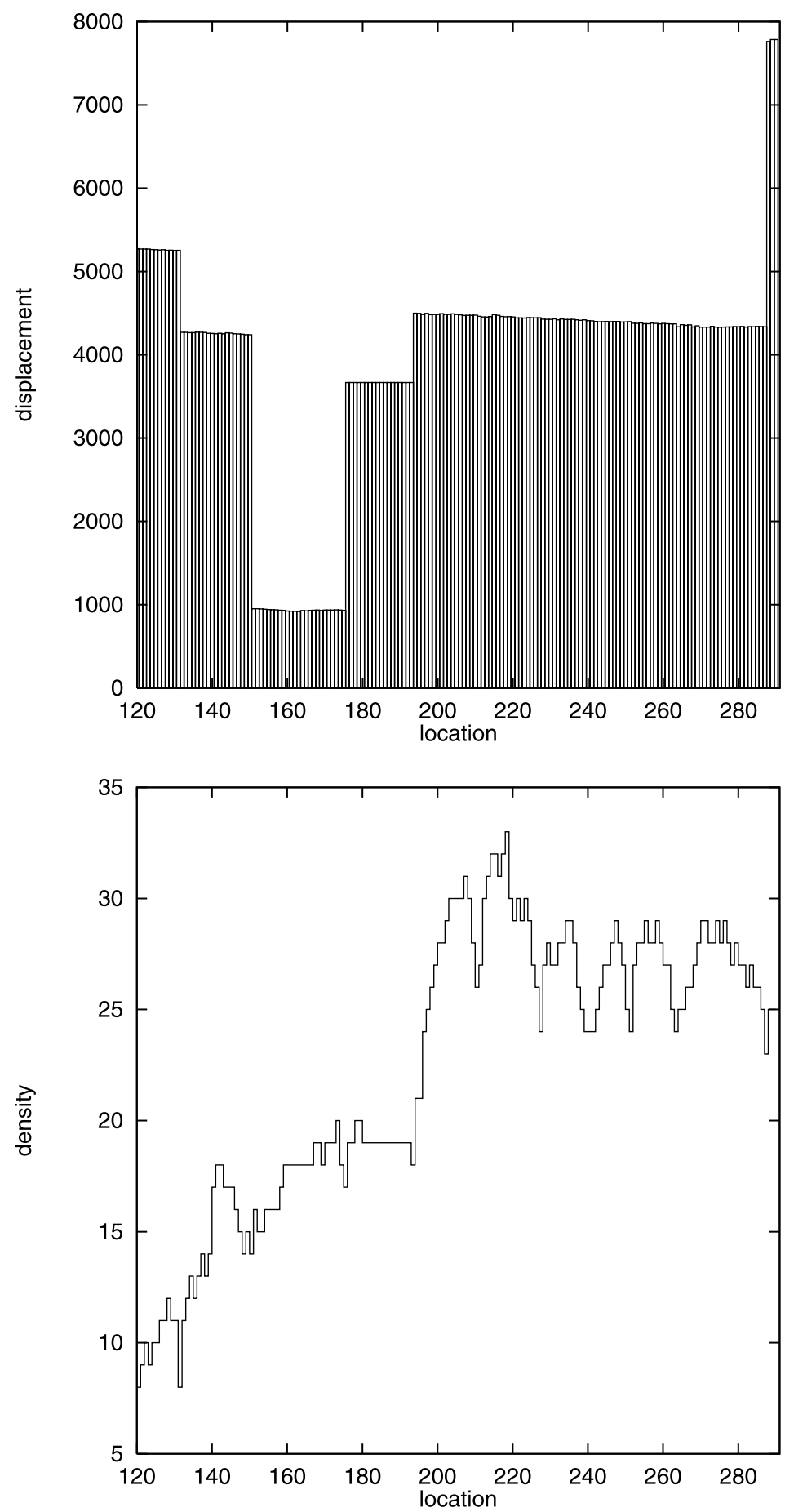

FIGURE 12 An example to show the correlation between the edges of plateaus and their corresponding density values in a placement for circuit s38584.

and the hybrid spectral method (SLPC2) of [1]. Using the RBLS/C technique, new best-known result for every benchmark circuit have been found. Table Isummarized the results. We also show the best results obtained by the RBLS technique only (without clustering) in the table. As the table shows, the effect of the clustering technique is large, particularly for the two large circuits, s38584 and s38417. Since Ref. [2] used a different version of s1423 we do not compare our result for s1423 with that of the SQ method.
To show CPU time, we made 10 runs for each test case and showed the best and worst results with the average running time in Table II. The CPU time to get a reasonable solution for the larger circuits (s38584 and s38417) seems promising: typically less than $2 \mathrm{~h}$ is required. For comparison, [2] reports about $8 \mathrm{~h}$ on a comparable machine for $\mathrm{s} 38417$.

We also compare the RBLS/C technique with the CLP technique in [3] on the same set of circuits. As did in [3], we also made 100 runs for each test circuit and show the 


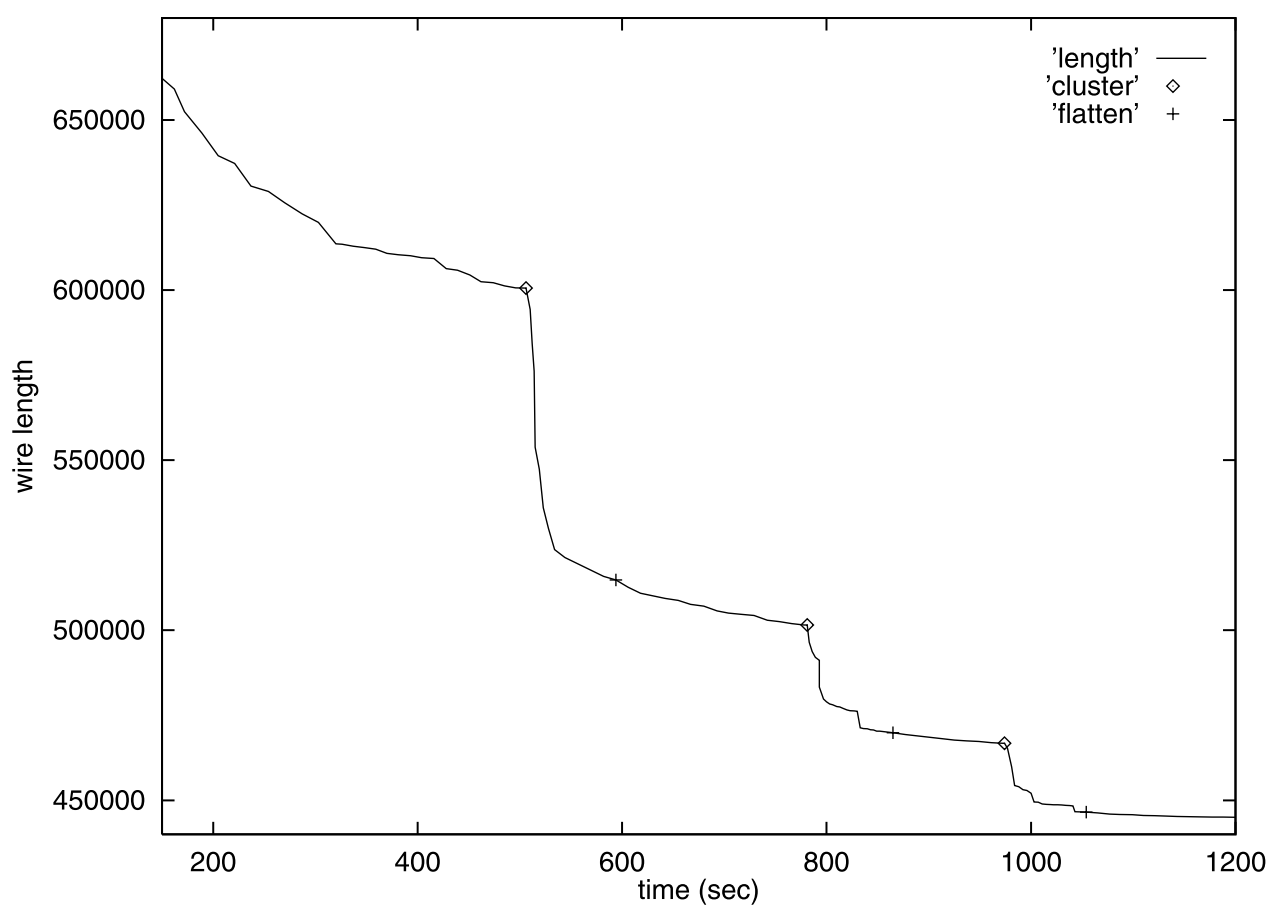

FIGURE 13 Effect of Dynamic Clustering. The figure shows part of a run for circuit s15850 where we toggle between flat and cluster mode. Before clustering, optimization of the flat placement has nearly converged.

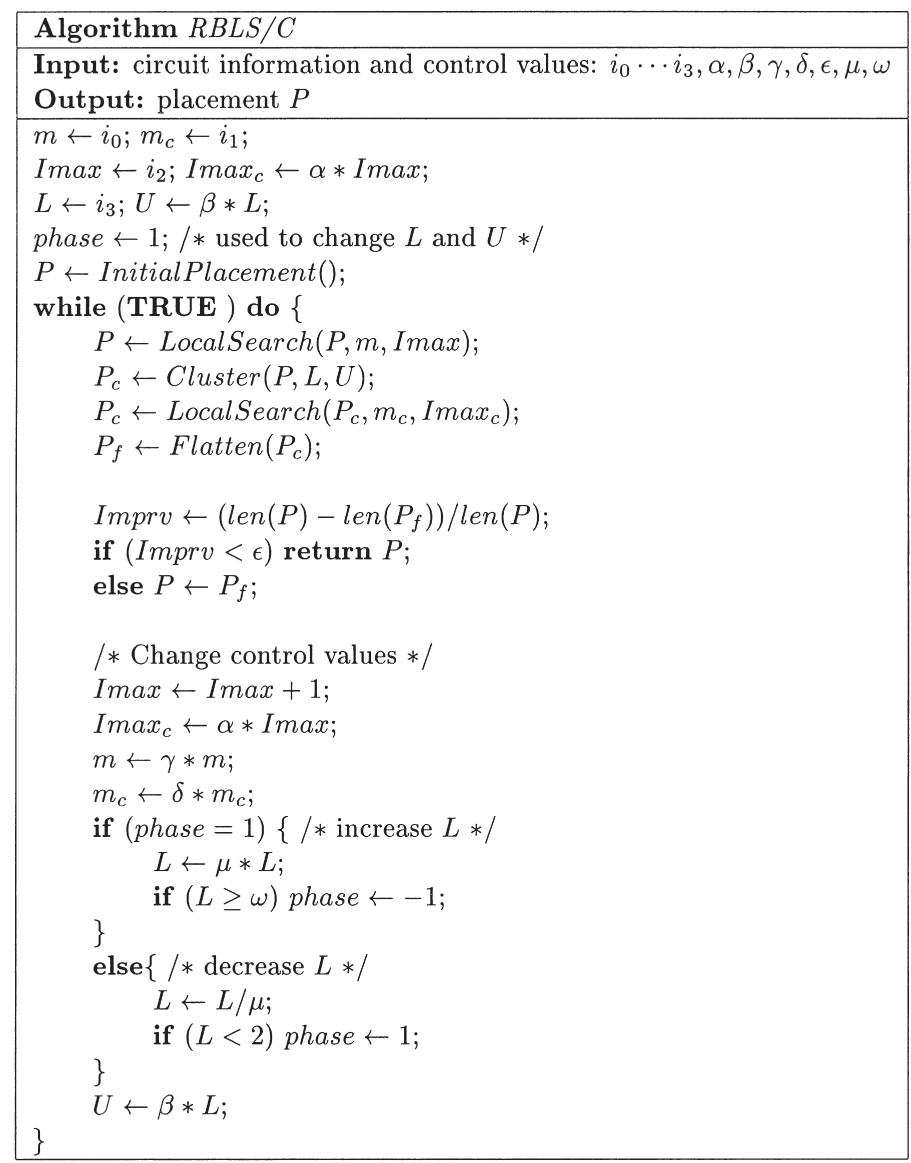

FIGURE 14 Overall procedure of the RBLS/C algorithm. 
TABLE I Best results compared with SLPC2 [1] and SQ [2] methods

\begin{tabular}{|c|c|c|c|c|c|c|c|c|}
\hline \multirow{2}{*}{ Ckts } & \multirow{2}{*}{ Nodes } & \multirow{2}{*}{ Nets } & \multicolumn{4}{|c|}{ Wire length } & \multicolumn{2}{|c|}{ (\%) Improv. over } \\
\hline & & & SLPC2 & SQ & RBLS & RBLS/C & SLPC2 & SQ \\
\hline s1423 & 619 & 538 & 9254 & - & 8776 & 8373 & 9.5 & - \\
\hline s9234 & 5866 & 5844 & 248,999 & 220,374 & 232,026 & 199,574 & 19.8 & 9.5 \\
\hline s13207 & 8772 & 8651 & 465,214 & 380,908 & 437,190 & 351,614 & 24.4 & 7.7 \\
\hline s15850 & 10,470 & 10,383 & 591,372 & 432,277 & 485,257 & 415,589 & 29.7 & 3.9 \\
\hline S35932 & 18,148 & 17,828 & 871,937 & 774,414 & 760,016 & 752,618 & 13.7 & 2.8 \\
\hline S38584 & 20,995 & 20,717 & $1,325,547$ & $1,275,551$ & $1,489,131$ & $1,238,139$ & 6.6 & 2.9 \\
\hline \multirow[t]{2}{*}{ S38417 } & 23,949 & 23,843 & $1,487,277$ & $1,254,195$ & $1,591,574$ & $1,158,677$ & 22.1 & 7.6 \\
\hline & & & Average & & & & 18.0 & 5.7 \\
\hline
\end{tabular}

TABLE II Best and worst results with average CPU time among 10 runs for each test case

\begin{tabular}{lrrr}
\hline & \multicolumn{2}{c}{ Wire length } & \\
\cline { 2 - 3 } Ckts & \multicolumn{1}{c}{ Best } & \multicolumn{1}{c}{ Worst } & Avg. CPU time (min) \\
\hline S1423 & 8726 & 9564 & 0.4 \\
S9234 & 207,928 & 223,876 & 22.5 \\
S13207 & 385,249 & 473,342 & 17.4 \\
S15850 & 452,488 & 495,277 & 22.2 \\
S35932 & 771,412 & 778,268 & 21.7 \\
S38584 & $1,282,056$ & $1,374,314$ & 94.4 \\
S38417 & $1,218,494$ & $1,511,268$ & 110.5 \\
\hline
\end{tabular}

relative wire length compared to the LESS technique [18]. In Tables III and IV, we show the results on circuits. The solutions obtained by RBLS/C technique are very comparable to those by CLP technique except for one circuit, CKT7. For the circuit CKT7, the wire length by RBLS/C is about $15 \%$ worse on average than that by CLP method.

\section{CONCLUSIONS}

Through a case-study of the linear placement problem, this paper provides new perspectives on each of three fundamental techniques in CAD/VLSI-constraint relaxation, local search, and clustering.

First, we propose a local search mechanism in which the neighborhood operator itself is based on constraint relaxation techniques popular in analytical placement. The result is a sophisticated neighborhood operator which enables very directed solution space exploration. The operator is based on a linear programming relaxation of the problem. We have devised an efficient algorithm to solve the linear programming formulation using networkflow techniques. The overall result is an optimization technique which explores the solution space with a more analytical and global view of the problem while maintaining the desirable features of the local search paradigm.

TABLE III Results compared with CLP [3] on smaller circuits

\begin{tabular}{|c|c|c|c|c|c|c|c|c|c|c|c|}
\hline \multirow[b]{2}{*}{ Ckts } & \multirow[b]{2}{*}{ Nodes } & \multirow[b]{2}{*}{ Nets } & \multirow[b]{2}{*}{ LESS [18] } & \multicolumn{4}{|c|}{ CLP } & \multicolumn{4}{|c|}{ RBLS/C } \\
\hline & & & & Min & Mean & Max & Std & Min & Mean & Max & Std \\
\hline Fig. 1.2 & 5 & 131 & 243 & 1.00 & 1.00 & 1.00 & 0.00 & 1.00 & 1.00 & 1.00 & 0.00 \\
\hline Fig. 1.4 & 5 & 31 & 43 & 1.00 & 1.00 & 1.00 & 0.00 & 1.00 & 1.00 & 1.00 & 0.00 \\
\hline Fig. 9a & 6 & 50 & 78 & 1.00 & 1.00 & 1.00 & 0.00 & 1.00 & 1.00 & 1.00 & 0.00 \\
\hline Fig. 8 & 9 & 21 & 50 & 1.00 & 1.00 & 1.00 & 0.00 & 1.00 & 1.00 & 1.00 & 0.00 \\
\hline Data III & 15 & 18 & 65 & 1.00 & 1.00 & 1.00 & 0.00 & 1.00 & 1.00 & 1.00 & 0.00 \\
\hline Data V & 29 & 37 & 220 & 0.99 & 1.00 & 1.06 & 0.01 & 0.95 & 0.96 & 1.01 & 0.02 \\
\hline Data VI & 48 & 48 & 337 & 0.99 & 1.01 & 1.06 & 0.01 & 0.99 & 1.00 & 1.01 & 0.00 \\
\hline Fig. 10 & 85 & 96 & 1009 & 0.99 & 1.02 & 1.10 & 0.02 & 0.98 & 1.02 & 1.11 & 0.03 \\
\hline
\end{tabular}

TABLE IV Results compared with CLP [3] on large circuits

\begin{tabular}{|c|c|c|c|c|c|c|c|c|c|c|c|}
\hline \multirow[b]{2}{*}{ Ckts } & \multirow[b]{2}{*}{ Nodes } & \multirow[b]{2}{*}{ Nets } & \multirow[b]{2}{*}{ LESS [18] } & \multicolumn{4}{|c|}{ CLP } & \multicolumn{4}{|c|}{ RBLS/C } \\
\hline & & & & Min & Mean & Max & Std & Min & Mean & Max & Std \\
\hline CKT1 & 60 & 75 & 608 & 1.00 & 1.02 & 1.04 & 0.01 & 1.00 & 1.00 & 1.02 & 0.007 \\
\hline CKT2 & 183 & 165 & 1230 & 1.00 & 1.04 & 1.18 & 0.04 & 1.00 & 1.01 & 1.05 & 0.010 \\
\hline CKT3 & 200 & 300 & 9119 & 0.99 & 1.01 & 1.04 & 0.01 & 0.99 & 0.99 & 1.02 & 0.005 \\
\hline CKT4 & 286 & 307 & 4031 & 0.88 & 0.93 & 1.02 & 0.03 & 0.89 & 0.94 & 0.99 & 0.024 \\
\hline CKT5 & 469 & 451 & 6346 & 0.90 & 0.94 & 1.04 & 0.02 & 0.90 & 0.91 & 0.91 & 0.001 \\
\hline CKT6 & 800 & 684 & 34,640 & 0.97 & 1.04 & 1.18 & 0.04 & 0.96 & 0.97 & 0.98 & 0.002 \\
\hline CKT7 & 3060 & 3123 & 156,300 & 0.71 & 0.76 & 0.86 & 0.04 & 0.78 & 0.87 & 0.93 & 0.045 \\
\hline
\end{tabular}


Second, we present a new dynamic clustering strategy for escaping local optima. The strategy is motivated by the studies which characterize local optima in the linear placement problem and their relation to circuit clusters. We have devised a simple visualization tool-the displacement graph-for characterizing the differences between two placements. By this tool, we have shown that a local optima typically have successfully found many appropriate clusters of cells, but that these clusters are not globally placed correctly. A local search algorithm working on the flat netlist is unlikely to uncover this structure and as a result it is usually very difficult to improve such solutions. However, via our dynamic clustering strategy based on the current linear placement, such global structure can often be revealed, allowing us to escape the local optima.

The experimental results prove the effectiveness of our RBLS/C algorithm, which incorporates both the relaxation-based local search and the dynamic clustering techniques, by generating new best known results using less CPU-time for each circuit on which Sato [2] and $\mathrm{Li}$ et al. [1] have tested.

A natural progression of this work is to adapt the techniques to the 2D case. An interesting visualization question is what is the 2D analogy of the displacement graph? Since there are 4 dimensions to consider $(x, y, \Delta x$ and $\Delta y$ ), creative use of color may be necessary to reveal 2D clustering information. More in-depth study of clustering techniques-including hierarchical is also a possibility. Finally, we also are generalizing the techniques for the timing-driven placement problem.

\section{References}

[1] Li, J., Lillis, J., Liu, L.-T. and Cheng, C.K. (1996) "New spectral linear placement and clustering approach", 33rd DAC, pp. 88-93.

[2] Sato, S. (1997) "Simulated quenching: new placement method for module generation", Proceedings of ICCAD (IEEE Computer Society Press, San Jose, CA) pp. 538-541.

[3] Saab, Y.G. (1996) "An improved linear placement algorithm using node compaction", IEEE Transactions on CAD of Integrated Circuits and systems 15(8), 952-958.

[4] Cheng, C.K. and Kuh, E.S. (1984) "Module placement based on resistive network optimization", IEEE Transactions on CAD, $218-225$.

[5] Sigl, G., Doll, K. and Johannes, F. (1991) "Analytical placement: a linear or a quadratic objective function", 28th ACM/IEEE DAC, pp. 427-432.

[6] Jackson, M.B. and Kuh, E.S. (1988) "Performance-driven placement of cell based ICs", 25th DAC, pp. 370-375.

[7] Frankle, J. and Karp, R.M. (1986) "Circuit placements and cost bounds by Eigenvector decomposition", Proceedings of ICCAD, pp. 414-417.

[8] Breuer, M.A. (1977) "A class of min-cut placement algorithms for the placement of standard cells", 14th DAC, pp. 284-290.
[9] Suaris, P.R. and Kedem, G. (1987) "Quadrisection: a new approach to standard cell layout", Proceedings of ICCAD, pp. 474-477.

[10] Sechen, C. and Sangiovanni-Vincentelli, A. (1986) "TimberWolf3.2: a new standard cell placement and global routing package", 23rd DAC, pp. 432-439.

[11] Mitra, D., Romeo, F. and Sangiovanni-Vincentelli, A. (1986) "Convergence and finite-time behavior of simulated annealing", Advances in Applied Probability, 747-771.

[12] Karypis, G., Aggarwal, R., Kumar, V. and Shekhar, S. (1997) "Multilevel hypergraph partitioning: applications in VLSI domain", 34th DAC, pp. 526-529.

[13] Alpert, C., Huang, J.H. and Kahng, A.B. (1997) "Multilevel circuit partitioning", 34th DAC, pp. 530-533.

[14] Picard, J.-C. and Ratliff, H.D. (1978) "A cut approach to the rectilinear distance facility location problem", Operations Research, pp. 422-433.

[15] Yang, H. and Wong, D.F. (1994) "Efficient network flow based mincut balanced partitioning", Proceedings of ICCAD (IEEE Computer Society Press, San Jose, CA) pp. 50-55.

[16] Liu, H. and Wong, D.F. (1997) "Network flow based multi-way partitioning with area and pin constraints", ISPD (ACM/IEEE, Napa Valley, CA) pp. 12-17.

[17] Alpert, C.J. and Kahng, A.B. (1994) "A general framework for vertex orderings, with applications to netlist clustering", Proceedings of ICCAD (IEEE Computer Society Press, San Jose, CA) pp. $63-69$

[18] Saab, Y.G. and Chen, C.-H. (1994) "An effective solution to the linear placement problem”, VLSI Design 2(2), 117-129.

\section{Authors' Biographies}

Sung-Woo Hur received the B.S. degree in Electronics from the Kyungpook National University in Korea, the M.S. degree in Computer Science from Korea Advanced Institute of Science and Technology in Korea and the Ph.D. degree in Electrical Engineering and Computer Science from the University of Illinois at Chicago. He is currently an associate professor in the department of Computer Engineering at Dong-A University in Korea. His research interests are CAD algorithms and computational geometry.

John Lillis received the B.S. degree in Computer Science from the University of Washington in 1989 and the M.S. and Ph.D. degrees in Computer Science from the University of California at San Diego in 1992 and 1996 respectively. From 1996 to 1997 he was an NSF postdoctoral visitor at the University of California at Berkeley. In 1997 he joined the University of Illinois at Chicago where he is currently an assistant professor in the department of Electrical Engineering and Computer Science. His research interests are in the areas of design automation for integrated circuits and combinatorial optimization. 

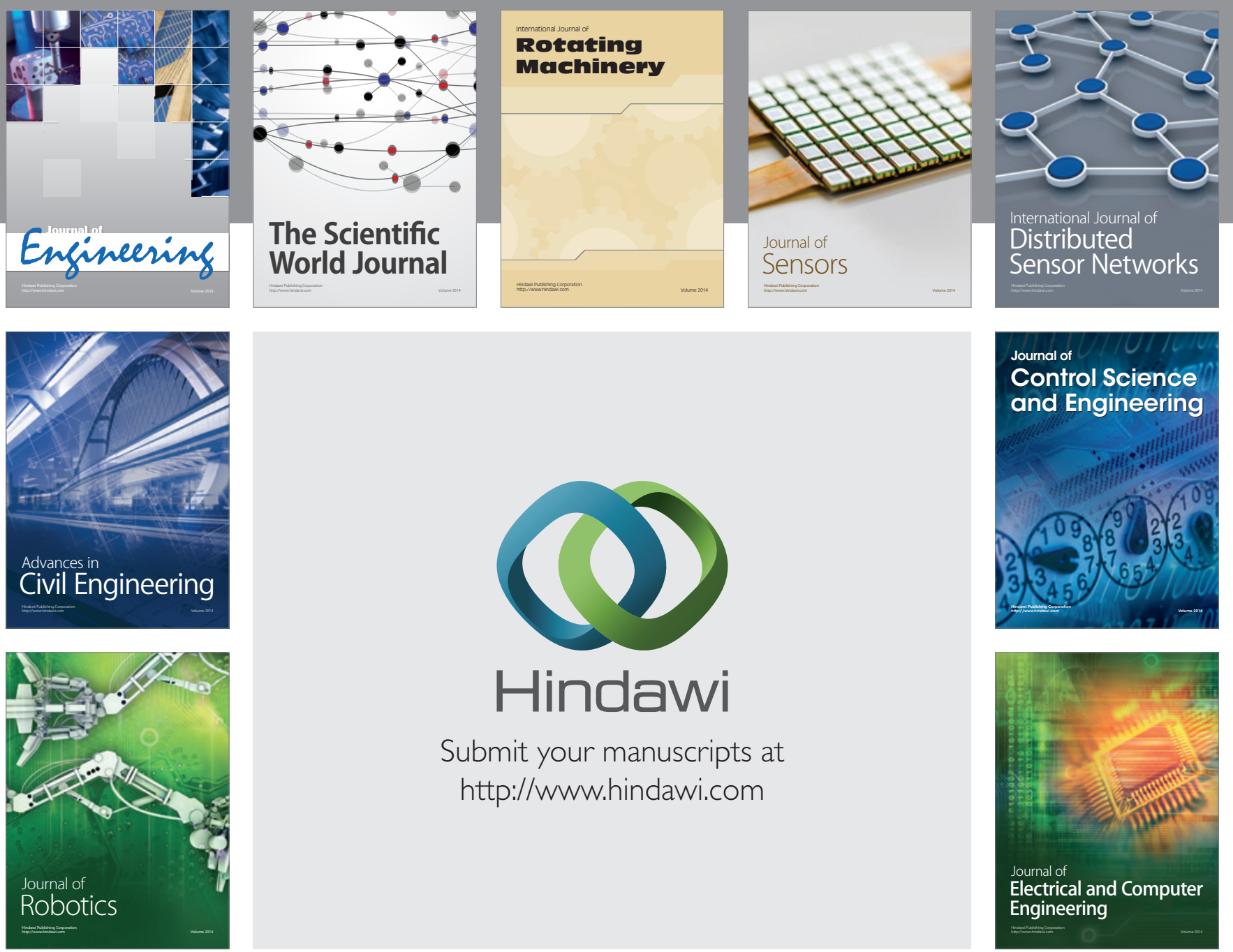

Submit your manuscripts at

http://www.hindawi.com
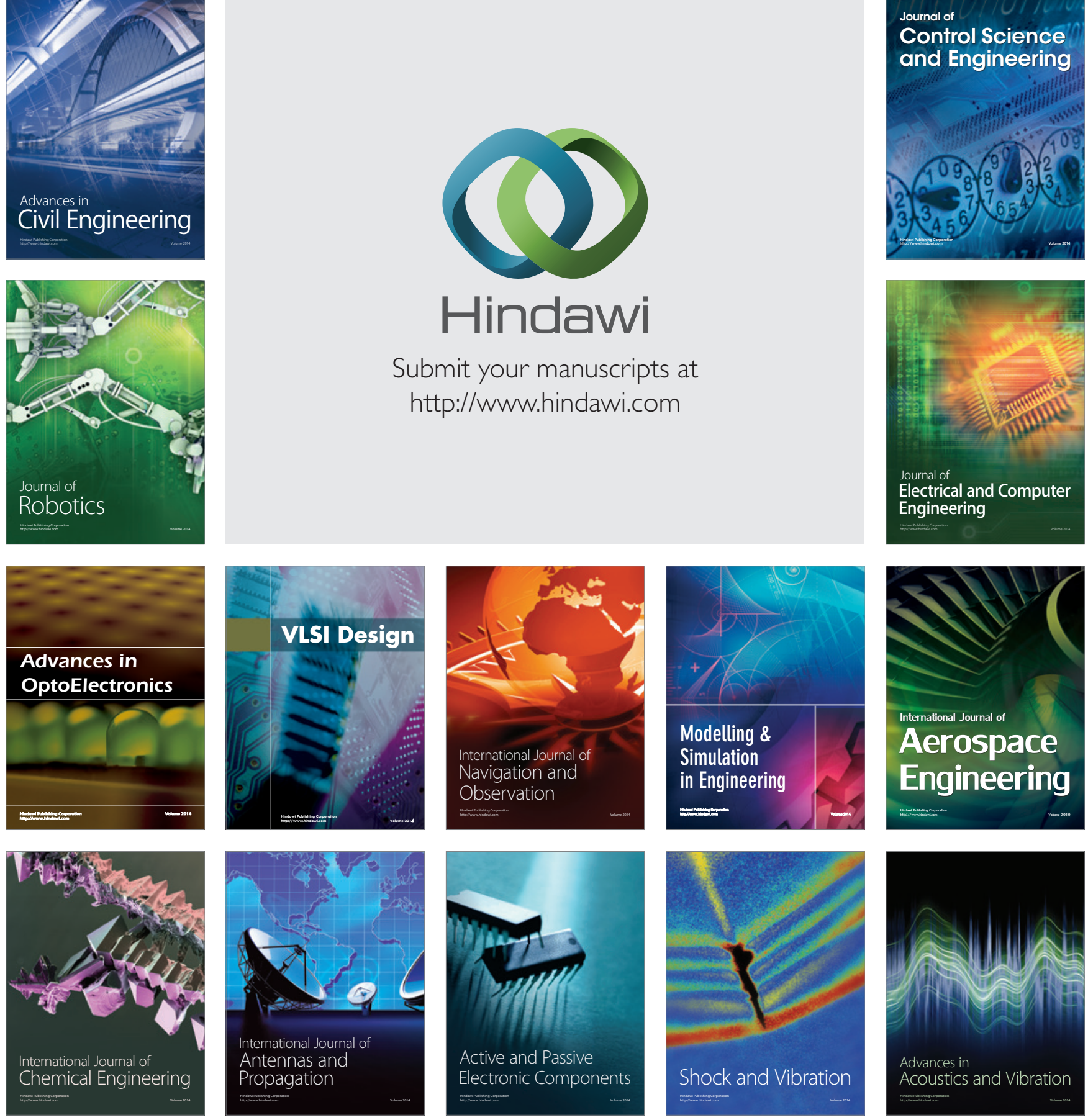